\title{
HIV-Infected Adolescent: A Case Report
}

\author{
Apezteguia Fernández Carolina Aurora ${ }^{1 *}$, Hernández Muniesa Belén ${ }^{1}$, Vicente Sánchez María del Pilar ${ }^{1}$, \\ Álvarez García Ana ${ }^{2}$, Ruiz Jiménez Marta², Ramos Amador José Tomás ${ }^{2}$ \\ ${ }^{1}$ Pharmacy Department, Getafe University Hospital, Getafe, Spain; ${ }^{2}$ Pediatric Infectious Diseases Unit, Getafe University Hospital, \\ Getafe, Spain. \\ E-mail: *carolina.apezteguia@salud.madrid.org
}

Received August 10 ${ }^{\text {th }}, 2011$; revised October $7^{\text {th }}, 2011$; accepted October $19^{\text {th }}, 2011$.

\begin{abstract}
We report the case of a Nigerian adolescent recently arrived to Spain, who presented at the emergency room with severe respiratory distress. She had been previously diagnosed of HIV-1 discontinuing antiretroviral therapy, what was hidden by the family. This case illustrates the difficulties in management and stigma in HIV-infected adolescents, particularly immigrants and the need to collect all the information available before starting antiretroviral therapy.
\end{abstract}

Keywords: HIV, Adolescent, Immigrant

\section{Introduction}

Human Immunodeficiency Virus (HIV) infection is one of the most important pandemic infections around the world. Perinatal transmission remains the main cause of HIV infection in paediatrics. The uses of highly active antiretroviral therapy (HAART) during pregnancy, antiretroviral prophylaxis for newborns and administration of intravenous zidovudine for pregnant women, have drastically reduced the rate of perinatally acquired HIV infection. Nevertheless, vertical transmission remains still high, especially in developing countries. Sub-Saharan Africa is the region most heavily affected by HIV. Almost $90 \%$ of HIV infected children younger than 15 years live in sub-Saharan Africa [1].

It is necessary to consider the particular features of HIV infection in paediatrics, clinical prognosis and evolution. Children have immature immune system, which makes them highly vulnerable to infections, such as HIV. An early diagnosis and treatment are essential to get a better prognosis and a lower incidence of AIDS progression. HAART is scaling up, but maintenance of adherence is a major barrier to complete control of viral replication. Herein, we present the case of a Nigerian girl recently arrived to Madrid, in whom the HIV diagnosis was not revealed and antiretroviral treatment had been discontinued.

\section{Case Report}

A 14 year-old Nigerian girl, 72 hours after arriving in Spain, was admitted to hospital, in September 2008, with a 2-day history of high fever, headache, cough, tachypnea and vomiting. No drug allergies were known and immunization schedule completed. Family history referred that mother died in Nigeria 5 years before by a car accident. HIV infection was suspected by physicians, because she came from a country with high HIV prevalence. In the emergency room, antibodies against HIV were positive. Her father and sister were unaware of her HIV-diagnosis, but referred a past history of malaria treated in Nigeria and some vitamines prescribed there. Her medical history from Nigeria was obtained one week later: in May 2007, our patient was diagnosed and classified in stage 2 of HIV infection (WHO), with $442 / \mu \mathrm{L}$ CD4 count. A fixed-dose combination of lamivudine, stavudine and nevirapine was started 6 months after diagnosis, (from December 2007 to June 2008). CD4 count fell to $168 / \mu \mathrm{L}$ probably because of poor adherence, and there was a disease progression until stage 3 (WHO).

At ER, physical examination revealed mild dehydration, malaise, tachycardia, tachypnea, slight drowsiness, bilateral neck lymphadenopathy, mild hepatomegaly and thrush. She was $148.2 \mathrm{~cm}$ in height and $35 \mathrm{Kg}$ in weight $(<\mathrm{P} 3)$. Other significant findings were hyponatremia $(\mathrm{Na}$ $=128 \mathrm{meq} / \mathrm{l})$, raised C-reactive protein $(>250 \mathrm{mg} / \mathrm{l})$ and procalcitonine of $26.1 \mathrm{ng} / \mathrm{ml}$. Immunoglobulins levels were increased: $3310 \mathrm{mg} / \mathrm{dl} \mathrm{IgG,} 214 \mathrm{mg} / \mathrm{dl} \mathrm{IgA}$ and 568 $\mathrm{mg} / \mathrm{dl}$ IgM. Cerebrospinal fluid (CSF) showed 58 cells (99\% mononuclears and $1 \%$ polinuclears), glucose 65 $\mathrm{mg} / \mathrm{dl}$, proteins $48 \mathrm{mg} / \mathrm{dl}$ and 0 red blood cells. Chest Xray showed bilateral alveolar infiltrates and brain CT 
scan was normal. Eye funduscopy was also normal. Echocardiogram revealed dilated myocardiopathy with preserved ejection fraction. HIV was confirmed by ELISA test and Western Blot (viral load: 133.000 copies/ml and CD4 count: $157 \mathrm{cel} / \mu \mathrm{L})$. HIV infection and bilateral pneumonia with alveolar pattern were the main diagnosis.

Microbiological findings showed the following results: blood thick smear and urine antigen test for malaria were negative. PCR blood for malaria was positive and in blood culture grew Streptococcus pneumoniae. Pneumonia by Streptococcus pneumoniae was confirmed and cefotaxime was continued for 10 days, remitting fever and dyspnea. She also received intravenous trimethoprim/ sulfamethoxazole for covering Pneumocystis jiroveci (PJ) pneumonia and Atovaquona-proguanile (Malarone ${ }^{\circledR}$ ) for Plasmodium falciparum malaria. Bronchoalveolar lavage showed negative results for Pneumocystis jiroveci, so intravenous trimethoprim/sulfamethoxazole was discontinued and switched to oral prophylactic doses. Parasite tests made for Cryptosporidium and Schistosoma were negative. Mantoux, gastric washing for culture and PCR for mycobacterium were also negative. Blood PCR for criptococcus and cerebrospinal fluid (CSF) PCR for pneumococcus and mycobaterium showed negative results. In the absence of microbiologial diagnosis, the increase of CSF cellularity was interpreted as possibly related to HIV-infection.

Infection symptoms improved and HAART was decided to start one week later, when genotypic resistance testing was available and patient and family were prepared to start treatment. HLA-B*5701 test showed negative result. The subtype of HIV-1 was G and the stage of infection was determined to be B3. All mutations detected (L10:I, V82:I, L89:M. V179:I) not conferred resistance to protease (PIs) and retrotranscriptase (RT) inhibitors. It was decided to use 3 new drugs in the new regimen: ddI $\mathrm{qd}+\mathrm{ABC} \mathrm{qd}+$ lopinavir/ritonavir bid after getting genotypic resistance testing and considering the previous history on antiretrovirals, because of the likelihood of selecting for resistance due to previous exposure.

The patient was discharged from hospital two weeks after admission, with antiretroviral treatment and oral prophylaxis against PJ. Clinical evolution and adherence follow-up was carried out by a multidisciplinary team (physician, psychologist and pharmacist). She had an excellent adherence and response to AR. The viral load became undetectable and the CD4 increased up to 617 (17\%) at 6 months of therapy. PCP prophylaxis was then discontinued. Twelve months after the initation of HAART, in the routine trimestral visit, the viral load went up to $2811 \mathrm{cop} / \mathrm{ml}$ (3.45 log) and CD4 count was 622 (19\%). In the hospital pharmacy, adherence was calculated through two indirect methods: dispensing record and valid questionnaire. Our patient referred having some difficulties taking lopinavir/ritonavir bid, but she did not recognize missing any dose. This medicationrelated problem was detected by compliance questionnaire and a simultaneously increase of viral load occurred (Figure 1). When adherence was improved and the viral load returned to $<50$ copies/ml, lopinavir/ritonavir was switched to atazanavir/ritonavir qd with good adherence and tolerance and good control of viral replication. 12 months after switching, she had undetectable viral load and CD4 > 500. Last CD4 was $953(32 \%)$ cells $/ \mu 1$.

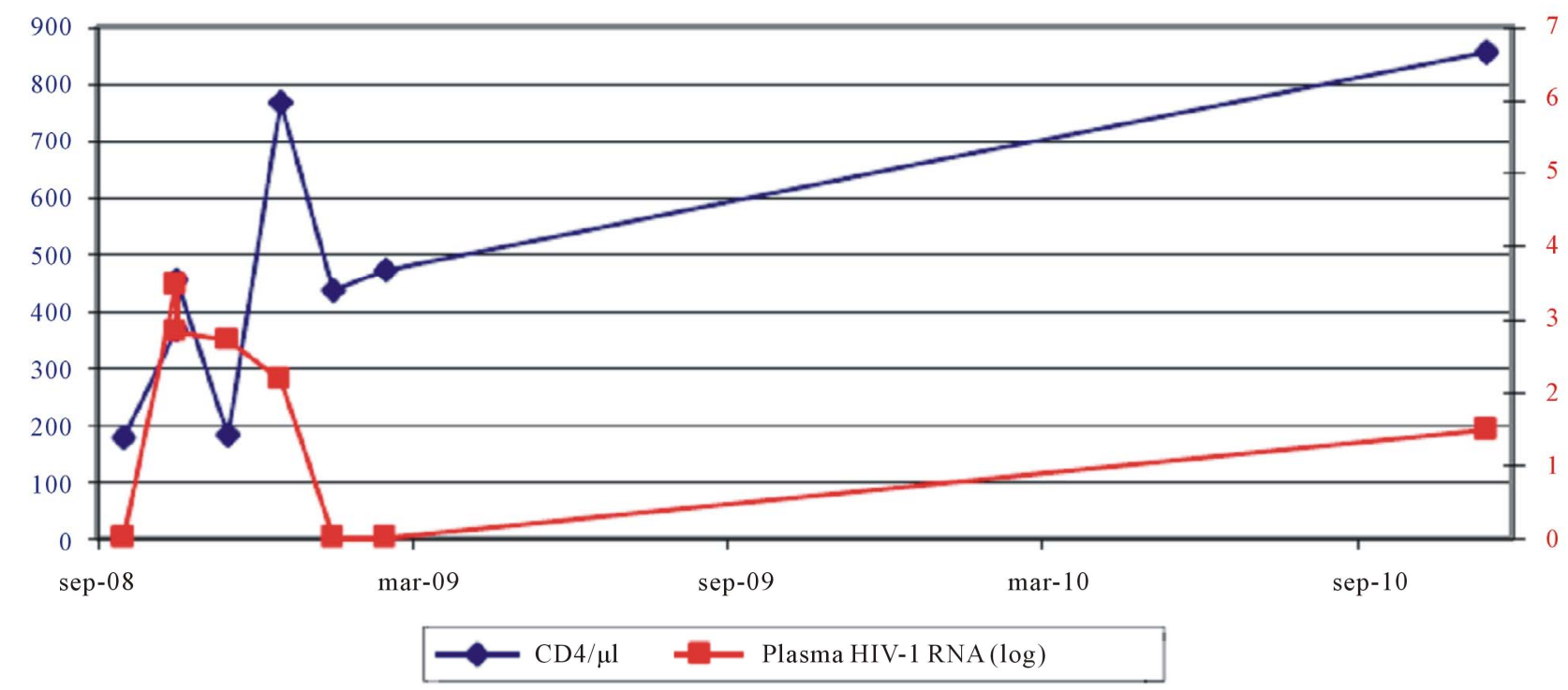

Figure 1. Clinical parameters evolution. 


\section{Discussion}

Currently, paediatric HIV infection has become a chronic disease with an excellent long term prognosis [2]. Adherence is critical in determining the degree of viral suppression achieved in response to antiretroviral therapy [3-5]. It is reported to be suboptimal among children and even worse among adolescents [6]. Suboptimal adherence can lead to subtherapeutic levels of antiretrovirals with both risk of development of drug resistence and virologic failure $[4,6]$. Factors, such as medication formulation, frequency of dosing, child age and psychosocial characteristics, have been associated with treatment compliance [7]. Monitoring adherence by questionnaires and counselling by a multidisciplinary team improve compliance and is helpful to detect and solve medication-related problems.

There is an additional problem among immigrant groups. It is needed to consider the social and cultural determinants of immigrant adolescents in the context of their cultural and social norms and the role of family relationships. All the cultural and social inhibitions may make the management difficult and knowledge of HIV and illustrate the difficulty of maintaining an optimal adherence [8]. In this case, our patient referred some difficulties taking lopinavir/ritonavir. It was detected by compliance questionnaire despite not recognizing missing any dose. Detecting such medication-related problem is especially difficult between immigrants and may be responsible of failure therapy and resistance. Antiretroviral drug resistance testing is recommended to be incorporated into patient management to help the choice of new regimens and considering the possibility of prior HAART in adolescent recently arrived to European countries hampering new treatment options. The choice of the antiretroviral regimen was difficult in this case. First her family did not recognise HIV-diagnosis. Starting new medications in unwell patients without full information (previous antiretroviral agents prescribed, resistance testing, TB diagnosis excluded and potential adverse drug effects) is risky. HIV infection, previous exposure to HAART and the likelihood of developing resistance due to prior therapy must be considered by physicians in every adolescent or child who come from an endemic HIV area. In our case, considering the previous history on antiretrovirals, it was decided to use ddI $+\mathrm{ABC}+$ lopinavir/ritonavir in order to introduce as many new antiretrovirals as possible [3,4]. The optimal moment and type of antiretrovirals to start therapy are critical and a complete information is crucial. Depending on previous exposure to antiretroviral drugs there must be an increased risk of therapy failure. That is why all the pharmacotherapy history must be collected before start- ing HAART even if there is no resistance at all.

It is also essential to consider that HIV-infected patients are susceptible for developing opportunistic infections and the additional risk for coming from an endemic region for malaria and TB. In Africa, TB is the most common pulmonary complication of HIV. It is very important to do a differential diagnosis between TB and other types of pneumonia. The CD4 count can provide information about the type of pulmonary disease to which the patient is susceptible [9]. Malaria and HIV infections often coexist in areas of the world where these diseases have the largest burden, particularly in sub-Saharan Africa [10]. HIV status and immunosuppression may be associated with an increased risk of susceptibility to malaria infection. Conversely, malaria increases HIV replication and declines in CD4 cell counts [11]. HIV and malaria should be considered together as a part of healthcare programs for both diseases in countries where their copresence favors an interaction with important consequences [12].

This case illustrates several aspects: the stigma and secrecy in HIV-infected patients, particularly immigrants. In this population, maintaining an optimal adherence may be difficult due to cultural and social inhibitions. HAART failure depends on adherence patient and compliance therapy is reported to be suboptimal among children and even worse among adolescents. The possibility of HIV infection, previous exposure to HAART and the likelihood of developing resistance due to prior therapy must be considered by physicians in every child who comes from a high prevalence HIV area. The need to collect all the information available before starting antiretroviral therapy and genotyping testing is critical, because there must be an increased risk of therapy failure when there was previous exposure to antiretroviral drugs, even if there is no genotypic drug resistance. Improving patient's knowledge about HIV-infection and HAART therapy, a close follow-up and detecting medicationrelated-problems, might also be important tools to increase medication compliance and achieve complete viralsuppression.

\section{REFERENCES}

[1] United Nations Children's Fund/World Health Organization (UNAIDS/WHO), "Status of the Global HIV Epidemic," Report on the Global AIDS Epidemic, 2008.

[2] E. Núñez Cuadros, M. J. Mellado Peña, M. Rivera Cuello, M. Penim Fernández, R. Piñeiro Pérez, M. GarcíaHortelano, M. J. Cilleruelo Ortega, J. Villota Arrieta and P. Martín-Fontelos, "Antiretroviral Drug Toxicity in $\mathrm{Hu}-$ man Immunodeficiency Virus Infected Children," An Pediatr (Barc), Vol. 68, No. 5, 2008, pp. 425-431. 
[3] PENTA Steering Committee, "PENTA 2009 Guidelines for the Use of Antiretroviral Therapy in Paediatric HIV-1 Infection," HIV Medicine, Vol. 10, No. 10, 2009, pp. 591613. doi:10.1111/j.1468-1293.2009.00759.x

[4] Centers for Disease Control and Prevention, "Guidelines for the Use of Antiretroviral Agents in Pediatric HIV Infection," 2010, pp. 1-219. http://aidsinfo.nih.gov/ContentFiles/PediatricGuidelines.pdf

[5] R. B. Van Dyke, S. Lee and G. M. Johnson, "Reported Adherence as a Determinant of Response to Highly Active Antiretroviral Therapy in Children Who Have Human Immunodeficiency Virus Infection," Pediatrics, Vol. 109, No. 4, 2002, p. 61.

doi:10.1542/peds.109.4.e61

[6] B. Brichard and D. Van der Linden, "Clinical Practice Treatment of HIV Infection in Children," European Journal of Pediatrics, Vol. 168, No. 4, 2009, pp. 387-392. doi:10.1007/s00431-008-0914-8

[7] D. A. Murphy, M. Sarr, S. J. Durako, A. B. Moscicki, C. M. Wilson and L. R. Muenz, "Adolescent Medicine HIV/AIDS Research Network. Barriers to HAART Adherence among Human Immunodeficiency Virus-Infected Adolescents," Archives of Pediatrics \& Adolescent Medicine, Vol. 157, No. 3, 2003, pp. 249-255.
[8] D. Oshi, S. Nakalema and L. Oshi, "Cultural and Social Aspects of HIV/AIDS Sex Education in Secondary Schools in Nigeria," Journal of Biosocial Science, Vol. 37, No. 2, 2005, pp. 175-183. doi:10.1017/S0021932004006820

[9] J. M. Wallace, N. I. Hansen and L. Lavange, "Respiratory Disease Trends in the Pulmonary Complications of HIV Infection Study Cohort," American Journal of Respiratory and Critical Care Medicine, Vol. 155, No. 1, 1997, pp. 72-80.

[10] K. Hewitt, R. Steketee, V. Mwapasa, J. Whitworth and N. French, "Interactions between HIV and Malaria in Nonpregnant Adults: Evidence and Implications," AIDS, Vol. 20, No. 16, 2006, pp. 1993-2004. doi:10.1097/01.aids.0000247572.95880.92

[11] J. Mermin, J. R. Lule and J. P. Ekwaru, "Association between Malaria and CD4 Cell Count Decline among Persons with HIV," Journal of Acquired Immune Deficiency Syndromes, Vol. 41, No. 1, 2006, pp. 129-130. doi:10.1097/01.qai.0000179427.11789.a7

[12] M. D. Herrero, P. Rivas, N. I. Rallón, G. Ramírez-Olivencia and S. Puente, "HIV and Malaria," AIDS Reviews, Vol. 9, 2007, pp. 88-98. 